\title{
Un brote de beriberi en una cárcel colombiana
}

\author{
Fernando de la Hoz' Ligia Pérez², Víctor A. Olano $^{3}$, Bernardo Buitrago ${ }^{4}$
}

\begin{abstract}
Resumen
Se realizó una investigación de campo en Leticia (Amazonas) con el fin de esclarecer la causa del fallecimiento de un joven recluso en la cárcel municipal. Después de revisar la evidencia clínica y epidemiológica, concluimos que la causa de muerte fue una insuficiencia cardiaca con gasto alto, causada muy probablemente por déficit de tiamina (beriberi húmedo). Se hallaron cuatro casos más de beriberi mixto con manifestaciones moderadas, que pasaron sin diagnóstico, dentro de los reclusos. La dieta suministrada regularmente a los internos solo cubría la mitad de los requerimientos diarios de tiamina para personas adultas expuestas a un clima tropical. Este es el segundo informe publicado recientemente en Colombia sobre casos de beriberi y debe servir para que se extremen la vigilancia y las medidas dietarias de control en poblaciones altamente expuestas tales como aquellas institucionalizadas.
\end{abstract}

\section{An outbreak of beri-beri in a Colombian jail}

A field-study was carried out in Leticia (Amazonas department) in order to clarify the cause of death of a young man imprisoned in the municipal jail. After reviewing the clinical and epidemiological evidence, it was concluded that the cause of death was a heart attack, very probably caused by thiamine deficiency (wet beri-beri). Another four cases of mixed beri-beri were found amongst the prisoners, these having moderate manifestations, which had not been diagnosed. The diet given regularly to those incarcellated covered only half of the daily thiamine requirement for an adult population exposed to a tropical climate. This is the second report published recently in Colombia concerning beri-beri and should serve to tighten-up supervision of dietary control measures amongst high-risk populations, such as those confined to institutions.

El beriberi debido a la deficiencia de tiamina es una enfermedad predominante en aquellas áreas donde la base de la alimentación es el arroz. Hay dos variedades principales de la enfermedad: 1) beriberi seco que afecta principalmente el sistema nervioso periférico y
2) beriberi húmedo que se presenta con edemas que pueden ser o no generalizados y falla cardiaca de gasto alto; sin embargo, lo más frecuente es la combinación de las dos formas $(1,2)$.

1 Centro Control de Enfermedades, Subdirección de Epidemiología y LNR, INS.

2 Programa de Control de Enfermedades Transmitidas por Vectores del departamento del Amazonas.

3 Laboratorio de Entomología, INS

4 (Q.E.P.D) Laboratorio de Patología, INS

Recibido para su publicación: 10 de agosto de 1998 - Aceptado para su publicación: 22 de diciembre de 1998 
La gran mayoría de los colombianos tienen como base alimentaria el arroz trillado (sin cáscara); sin embargo, sólo hay un informe reciente de un brote de beriberi en una guarnición militar y éste que describimos a continuación (3).

Durante la primera semana de julio de 1994, el Servicio de Epidemiología Aplicada (SEA) del Instituto Nacional de Salud (INS) fue notificado acerca del fallecimiento de un recluso en la cárcel municipal de Leticia, capital del departamento de Amazonas. La causa del fallecimiento fue informada inicialmente como dengue hemorrágico o fiebre amarilla, pero, debido a que no se conocía en Leticia la presencia del vector urbano, Aedes aegypti, se decidió realizar una investigación de campo colaborativa entre el Servicio Seccional de Salud del Amazonas y el INS.

\section{Métodos}

Se realizó una investigación de brote, combinando la búsqueda de pacientes sospechosos de tener la misma enfermedad con encuestas entomológicas y con la búsqueda activa de casos sospechosos en la comunidad. El paciente tenía dos expedientes clínicos: uno pertenecía al momento cuando fue hospitalizado con el cuadro agudo y el otro estaba archivado en el departamento médico de la cárcel. Se revisó cuidadosamente la historia clínica hospitalaria del paciente, la cual había sido diligenciada por los médicos rurales del hospital de Leticia. Asimismo, se revisó la historia médica del paciente durante su estadía en la cárcel y se anotaron todos los hallazgos clínicos y paraclínicos relevantes para la investigación.

Se realizó una encuesta entomológica en la cárcel y en un radio de dos cuadras alrededor de ella con el fin de detectar la presencia de $A$. aegypti $u$ otros potenciales vectores de dengue o fiebre amarilla. Igualmente, se practicaron pruebas serológicas (IgM antidengue) a todas las personas que habían consultado por fiebre en el último mes en el hospital de Leticia.

Se aplicó una encuesta al resto de los reclusos con el fin de identificar otros casos sos- pechosos de padecer el mismo cuadro del paciente fallecido. Se hizo una definición de caso con el fin de identificar a todos los reclusos que durante el último año hubieran presentado cuadros sospechosos de déficit de tiamina. Se consideró como caso sospechoso de beriberi a todos aquellos reclusos que, durante el último año, hubieran presentado edema de miembros inferiores y parestesias en bota. Como caso confirmado, se definieron aquellos casos sospechosos que hubieran mejorado con un suplemento de tiamina.

Se revisó la dieta semanal que recibían los reclusos tanto la informada por el director del penal como también interrogando a algunos reclusos acerca de los alimentos consumidos durante la semana. Es de aclarar que el penal tiene una dietista contratada quien es la encargada de elaborar el menú. Por tanto, se cuenta con un documento escrito donde está pormenorizado la cantidad y el tipo de alimento que deben ser dados a los reclusos día por día durante una semana. A una muestra de los internos $(n=10)$ se les preguntó por el tipo de alimento que consumían, usando un formato de tipo entrevista. Especialmente, se indagó por la frecuencia con que ingerían ciertos alimentos de alto contenido en vitamina B como huevos o granos secos. También se preguntó por la frecuencia diaria con que ingerían carbo-hidratos en la dieta y la cantidad; se hizo énfasis en la cantidad de papas, yuca, pastas y arroz que consumían diariamente. Luego, se elaboró la tabla de alimentos que consumían más frecuentemente durante la semana y se calculó su contenido de tiamina usando para ello un programa de computadora diseñado para tal fin por Software Corporation (4).

\section{Pacientes y observaciones}

El caso índice fue un paciente de 24 años de edad, perteneciente a la etnia huitoto, natural de Puerto Arturo y procedente de la cárcel de Leticia quien consultó por un cuadro de casi un mes de evolución consistente en diarrea, edema de miembros inferiores, astenia, adinamia, anorexia, vómito alimentario, coluria y dolor 
abdominal en hipocondrio derecho y epigastrio. De acuerdo con la historia clínica, el paciente estaba afebril en el examen físico de ingreso, muy pálido, con tinte ligeramente ictérico en escleras, taquicárdico (FC 110/min), pero con la presión arterial en límites normales (110/60). Había buena ventilación pulmonar y se palpaba una hepatomegalia dolorosa a $3 \mathrm{~cm}$ por debajo del reborde costal; no se identificaron otras viscero-megalias. El diagnóstico inicial fue de abdomen agudo secundario a una hepatitis de etiología desconocida o a una pancreatitis. Los exámenes para hepatitis $\mathrm{B}$ fueron negativos al igual que la prueba de VIH.

Inicialmente, el paciente fue manejado como una pancreatitis con sonda nasogástrica, ranitidina intravenosa y meperidina. Unas horas más tarde, el paciente empezó a presentar deterioro de su estado neurológico con delirio, hipoactividad e hiporreflexia; además, se observó cianosis distal y una disminución importante del volumen urinario. En ese momento, los médicos tratantes pensaron en la posibilidad de malaria cerebral y ordenaron unos exámenes de hemoparásitos los cuales fueron negativos. Posteriormente, se cambió el diagnostico a sepsis bacteriana y se inició antibioticoterapia, furosemida, dopamina y una dosis rápida de glucosa al $10 \%$ ya que el paciente presentaba una glicemia de $51 \mathrm{mg} \%$. Un parcial de orina que se practicó era marcadamente patológico ya que se observó cilindruria, hematuria, leucocituria y proteinuria. Pese a todos los esfuerzos terapéuticos disponibles en el hospital de Leticia, el paciente entró en coma y falla renal por lo que falleció a las 48 horas de haber ingresado. El diagnostico clínico de la causa de muerte fue "hipoxia tisular debida a falla multisistémica".

La descripción microscópica de la autopsia indicaba que los órganos abdominales presentaban una fuerte coloración amarilla y se notaba una marcada congestión pulmonar. La evaluación microscópica indicaba una severa congestión vascular de todos los órganos estudiados y, en el corazón, se notaba una marcada hipertrofia de las miofibrillas $y$, especialmente, de sus núcleos que aparecían hipercromáticos y con mucha variabilidad morfológica de su estructura. En el hígado, se observaba congestión severa con necrosis acidófila hepatocitaria de tipo anóxico, debido a una insuficiencia cardiaca muy severa.

La historia médica anterior del paciente, desde su entrada a la cárcel, indicaba que a los 5 meses de estar detenido, en octubre de 1993, empezó a presentar edema y parestesias en bota, de ambas piernas; fue manejado con furosemida $y$, al parecer, mejoró pero, posteriormente, en febrero y marzo de 1994, nuevamente consultó por un cuadro de edema y parestesias de miembros inferiores. En todas las ocasiones en que consultó, se encontró taquicardia en reposo (>100 pulsaciones/min). Los exámenes de proteínas totales y de función renal practicados en esa época dieron resultados dentro de lo normal.

Usando la definición de caso antes descrita, logramos identificar dos personas más que habían presentado sintomatología parecida. Uno de ellos era un hombre peruano de 31 años, procedente de Lima, quien tenía 3 años de estar encarcelado y, en enero de 1994, empezó a presentar edema de miembros inferiores y parestesias. Consultó al médico de la cárcel y recibió un tratamiento parenteral con vitaminas del complejo $B$, después de lo cual mejoró dramáticamente. El otro caso era un hombre de 24 años de edad, procedente de Puerto Asís (Putumayo), quien tenía 18 meses de estar detenido y que, en abril de 1994, presentó igual sintomatología que el anterior paciente. Fue tratado con complejo B también y se mejoró rápidamente de su sintomatología. Otros dos internos habían presentado igual sintomatología, pero ya habían sido dejados en libertad y no fue posible localizarlos. Todos los pacientes recibieron tratamiento antes de que se realizara la encuesta.

El cuadro 1 muestra la dieta regular que recibían los reclusos y la cantidad de tiamina que suministraba cada alimento. A cada uno de los alimentos se le calculó la cantidad promedio de tiamina que suministraba. Se puede apreciar 
Cuadro 1. Descripción de la dieta básica de los reclusos y su contenido de tiamina.

\begin{tabular}{lcc}
\hline Alimento & Frecuencia & Contenido de tiamina \\
\hline Chocolate & diaria & 0,06 \\
Arroz & diaria & 0,10 \\
Pan & diaria & 0,24 \\
Papa & diaria & 0,11 \\
Sopa de pasta & diaria & 0,10 \\
Pasta & 2-3 por semana & 0,26 \\
Plátano & diaria & 0,11 \\
Pescado & 2-3 por semana & 0,06 \\
Carne & semanal & 0,10 \\
Huevo & semanal & 0,10 \\
Fríjoles & semanal & 0,60 \\
Lentejas & semanal & 0,70 \\
\hline
\end{tabular}

que los alimentos consumidos más frecuentemente por los reclusos sólo suministraban $0,98 \mathrm{mg}$ de tiamina por día, mientras que los requerimientos diarios para un adulto en climas tropicales es del orden de 1,5 mg y más si hace una actividad moderada o fuerte. Por otra parte, en el cuadro 1 también se puede observar que los reclusos consumían diariamente diversos alimentos ricos en carbohidratos tales como plátano, arroz, pastas y papas, lo cual incrementa también las necesidades de tiamina.

La encuesta entomológica del penal reveló gran cantidad de criaderos de larvas, pero, ninguno de ellos fue positivo para $A$. aegypti; tampoco se encontraron adultos pertenecientes a este género. Se inspeccionaron otros criaderos en la ciudad y todos fueron negativos para vectores de fiebre amarilla o dengue.

Se tomaron 15 muestras serológicas a personas que habían tenido fiebre en el último mes y habían consultado al hospital de Leticia. Estas muestras fueron todas negativas para $\operatorname{lgM}$ antidengue $\mathrm{o} \lg G$ antidengue.

\section{Discusión}

Decidimos clasificar el caso índice como beriberi húmedo teniendo en cuenta los siguientes criterios clínicos y epidemiológicos: 1) todos los pacientes estaban sometidos a una dieta deficiente en tiamina;
2) cuando los síntomas empezaron, los pacientes estaban detenidos y, por tanto, sometidos a una dieta deficitaria en tiamina, un período de tiempo compatible con el período de incubación del beriberi (80 a 100 días) (1);

3) no había evidencia de enfermedades infecciosas que también pudieran ocasionar insuficiencia cardiaca por sepsis, y

4) otros casos dentro de la cárcel tuvieron sintomatología inicial semejante a la que presentó el caso índice y mejoraron en forma dramática al ser tratados con complejo B. Estos pacientes podrían ser definidos como de beriberi mixto.

La sospecha de que pudiera tratarse de un caso de muerte por deficiencia nutricional surgió al comparar los hallazgos físicos y patológicos del paciente con los encontrados en un brote de beriberi en la Escuela Naval de Cartagena donde un número significativamente mayor de soldados estuvieron afectados y también con varios casos fatales. Sin embargo, vale la pena resaltar que estos cuadros se pueden confundir fácilmente con cuadros de sepsis o de infecciones por enterovirus. También, se debe tener en cuenta que las intoxicaciones por metales pesados, como mercurio y talio, pueden simular los síntomas del beriberi, especialmente en la afección del sistema nervioso periférico. Sin embargo, la presencia concomitante de edema y parestesias debe hacer sospechar la deficiencia de tiamina en climas tropicales (3).

Podría argumentarse que habiendo pruebas enzimáticas de laboratorio para detectar el déficit de tiamina, tal como la actividad de la transcetolasa eritrocitaria, se debería recurrir a ellas para el diagnóstico definitivo. Sin embargo, estas pruebas no están fácilmente disponibles en el país y la mayoría de los investigadores y clínicos afirma que el diagnóstico se debe hacer por clínica y epidemiología (5). Por otra parte, el examen histopatológico de una muestra de tejido cardíaco del paciente recientemente fallecido, mostró un patrón anatomopatológico muy compatible con la afección miocárdica del beriberi húmedo. 
El paciente no tenía familiares en Leticia y, por tanto, no tenía opción de cambiar el menú de la prisión, cosa que sí sucede con otros reclusos que, por tener familiares en la misma ciudad, tienen acceso frecuente a otros tipos de alimentos diferentes a los que se proveen en el penal. Además, la dieta de los indígenas del área es también muy rica en carbohidratos y pobre en tiamina, lo cual probablemente nos habla de que el paciente podría haber tenido ya niveles bajos de esta vitamina antes de ingresar a la prisión, déficit que se agravó con su estadía en el penal.

Algunos aspectos del manejo del caso fatal pudieron agravar el pronóstico del paciente, por ejemplo, la administración de glucosa intravenosa puede desencadenar el cuadro agudo de beriberi cardíaco, mientras que la furosemida aumenta la excreción urinaria de tiamina depletando aún más los escasos depósitos de vitamina B1 que pudiera tener el paciente $(6,7)$.

Algunos de los factores de riesgo que se asocian con el beriberi son: el alcoholismo, la drogadicción, la alimentación parenteral, el vivir en cuarteles, prisiones o albergues, la infección por VIH y, en recién nacidos, el nacer de madres con déficit de tiamina (retardo de crecimiento intrauterino).

En el brote descrito en este trabajo, ninguno de los pacientes tenía antecedentes importantes de alcoholismo ni de drogadicción, pero, la dieta que recibían era totalmente inadecuada para suplir las necesidades de tiamina de un adulto normal. Estos requerimientos son de $1,5 \mathrm{mg}$ diarios, pero, cuando las dietas son muy ricas en carbohidratos como era el caso de la suministrada por las autoridades de la cárcel, entonces los requerimientos de tiamina son mayores, dado que esta vitamina es un cofactor importante en el metabolismo de los azúcares. Además, el ejercicio físico aumenta también los requerimientos de vitamina B1. Pudimos documentar que el caso índice de este estudio fue un practicante asiduo de fútbol durante los primeros meses de su estadía en la cárcel de Leticia; aparentemente, jugaba más de dos horas al día cada día hasta el momento cuando empezó a presentar el edema de miembros inferiores. Este ejercicio intenso, unido a la dieta inadecuada, pudo precipitar la caída en los niveles de tiamina $y$, posteriormente, el cuadro de beriberi húmedo.

Algunos autores creen que la presencia de beriberi húmedo o seco está influida por la relación entre la dieta y el ejercicio. Esto significa que si los pacientes consumen una dieta muy rica en carbohidratos y, además, hacen mucho ejercicio, se favorecerá la presentación de las manifestaciones cardiovasculares del déficit de tiamina, mientras que si se da lo contrario, predomina el cuadro neurológico (6).

Varias enfermedades se deben tener en cuenta como diagnósticos diferenciales del beriberi. En la etapa donde predominan los síntomas neurológicos periféricos, es importante descartar la intoxicación por metales pesados, especialmente talio; mientras que, en la fase de falla cardíaca, es la sepsis uno de los diagnósticos más importantes que se deben descartar, especialmente en pacientes jóvenes sin ninguna historia de problemas cardíacos hasta el momento $(8,9)$.

Existen muy pocos estudios sobre beriberi en Latinoamérica; una revisión hecha al índice electrónico de publicaciones latinoamericanas (LILACS) reveló que se han producido sólo 7 publicaciones sobre el tema en los últimos 5 años y todas son descripciones de pacientes con beriberi cardíaco de Brasil. En un estudio reciente de Brasil, se describen dos casos de polineuropatía asociada con déficit de tiamina en dos individuos jóvenes pertenecientes a la etnia xavante en la Amazonia brasileña donde la dieta básica es a base de arroz procesado. Esto puede sugerir que, en estas regiones tropicales, una alta proporción de la población podría estar expuesta a dietas deficientes de tiamina con el consiguiente riesgo de beriberi $(10,11)$.

Dadas las consideraciones anteriores, llama la atención que no se informen más casos de 
beriberi en las zonas tropicales del país. Este es un aspecto digno de reflexión, mirando los resultados de este y de otros estudios publicados en el país. Una explicacion podría ser la automedicación de vitaminas que es una conducta frecuente en todo el país y en todas las capas sociales, pero, especialmente entre los estratos socioeconómicos menos favorecidos. Esta autoformulación podría ser un factor protector contra las enfermedades carenciales en algunas zonas del país. Otra razón muy plausible para que ello no suceda es la falta de diagnóstico de esta enfermedad que muchos médicos consideran desaparecida desde hace mucho tiempo. Las últimas descripciones de beriberi entre nosotros, probablemente datan de la época de la guerra con Perú cuando precisamente los casos se presentaron entre soldados destacados en la Amazonia.

Esta investigación de campo muestra la importancia de integrar ciencias como la entomología, la clínica y la patología en la labor de confirmar casos de difícil diagnóstico en áreas tropicales. Asimismo, debe servir para alertar a la comunidad médica acerca de la presentación de esta enfermedad en grupos de alto riesgo para deficiencias nutricionales, dado que la falla cardíaca del beriberi evoluciona favorablemente a las pocas horas de haberse administrado intravenosamente un bolo de vitamina B1 (50-100 mg) en adición al tratamiento digitálico. Por tanto, se debe tener en cuenta siempre este diagnostico cuando se esté en presencia de personas jóvenes con falla cardíaca que no responde al tratamiento convencional.

La adición de tiamina a la dieta de poblaciones institucionalizadas debería prevenir la aparición de estos casos agudos que tienen una alta letalidad debido especialmente al desconocimiento de esta patología por parte de los médicos tratantes.

\section{Referencias}

1. Rolfe $\mathbf{M}$, Walger R, Samba K, Cham K. Urban beriberi in the Gambia, West Africa. Tr Roy Soc $\mathrm{Tr}$ Med Hyg 1993;87:114-5.

2. Vélez H. Deficiencia de tiamina y beriberi. En: Gastroenterología, hepatología y nutrición. Serie Fundamentos de Medicina. Medellín: ClB; 1990.p.581-2

3. Martínez M, Román G, de la Hoz F, Podlesky E, et al. Estudio clínico y epidemiológico de un brote de beriberi húmedo en Cartagena de Indias, Colombia 1992-1993. Biomédica 1996;16(1):41-51.

4. Software Corporation. Diet balance nutridata Software Corporation, 1988.

5. Valyasevi A, Munro H. Beriberi, pellagra and scurvy. En: Geographical and tropical medicine. Willey Liss Inc.; 1993.p.1051-6.

6. Wilson J. Vitamin deficiency and excess. En: Harrison's principles of internal medicine. 13th. edition. Mc Graw Hill Inc.; 1994.p.472-80.

7. Seligman H, Halkin H, Rauchfleish B, et al. Thiamin deficiency in patients with congestive heart failure receiving longterm furosemide therapy: a pilot study. Am J Med 1991;91:151-5.

8. Cavanagh J. What have we learnd from Graham Frederick Young? Reflections on the mechanism of thalium neurotoxicity. Neuropathol Appl Neurobiol 1991;17:3-9.

9. Román G. Nutrictional disorders. En: Windebank $A J$, editor. Polineuropathy due to nutritional deficiency and alcoholism. Third edition. WB Saunders and Co.; 1993.p.1310-7.

10. Blacher C, Barbisan J. Beriberi cardiaco: relato de um caso da forma fulminante (shoshin) e revisao de literatura. Rev AMRIGS 1985;29(2):136-41.

11. Viera Filho J, Oliveira AS, da Silva MR, Amaral AL, Schultz RR. Polineuropathy deficiency among Xavante indians. Rev Assoc Med Bras 1997;43(1): 82-8. 